\title{
Efficacy and safety of brinzolamide/timolol fixed combination compared with timolol in Japanese patients with open-angle glaucoma or ocular hypertension
}

Keiji Yoshikawa'

Jun Kozaki

Hidetaka Maeda ${ }^{3}$

'Yoshikawa Eye Clinic, Tokyo, Japan;

${ }^{2}$ Kozaki Eye Clinic, Osaka, Japan;

${ }^{3}$ Maeda Eye Clinic, Osaka, Japan
Correspondence: Keiji Yoshikawa

I-3-I Nakamachi Machida-shi Tokyo, Japan

Tel +8I 042739078 I

$\mathrm{Fax}+810427390782$

Email keiyomd@ark.ocn.ne.jp
This article was published in the following Dove Press journal:

Clinical Ophthalmology

10 February 2014

Number of times this article has been viewed

Purpose: To evaluate the efficacy and safety of brinzolamide $1 \%$ /timolol $0.5 \%$ fixedcombination (BRINZ/TIM-FC) therapy compared with timolol $0.5 \%$ (TIM) monotherapy in Japanese patients with open-angle glaucoma or ocular hypertension.

Methods: This randomized, double-masked, multicenter study included Japanese patients aged $\geq 20$ years. Patients were treated during a 4-week observation period with TIM monotherapy in advance of randomization to treatment with topical BRINZ/TIM-FC or TIM monotherapy twice daily for 8 weeks. The primary endpoint was mean reduction in intraocular pressure (IOP) from baseline to week 8 at 2 hours postinstillation. Adverse events (AEs) were recorded at each visit.

Results: A total of 301 patients (BRINZ/TIM-FC, $n=150$; TIM, $n=151$; age [mean \pm standard deviation], $61 \pm 13$ years) were enrolled. Mean IOP reductions from baseline were greater with BRINZ/TIM-FC than with TIM at weeks 4 and 8 at 0 and 2 hours postinstillation (all $P \leq 0.0001$ ), with mean reductions of $-3.2 \mathrm{mmHg}$ with BRINZ/TIM-FC and $-1.4 \mathrm{mmHg}$ with TIM at week 8 , 2 hours postinstillation. Although AEs were observed in 19\% of all patients (BRINZ/TIM-FC, $20 \%$; TIM, 19\%), all AEs were mild or moderate.

Conclusion: BRINZ/TIM-FC therapy was associated with significantly greater reductions in IOP compared with TIM, and it was well tolerated in Japanese patients with open-angle glaucoma or ocular hypertension.

Keywords: Azarga, intraocular pressure, carbonic anhydrase inhibitor, beta blocker

\section{Introduction}

If untreated or poorly managed, glaucoma often leads to progressive visual field defects that can progress to blindness. ${ }^{1}$ Even in its early stages, glaucoma poses a considerable economic burden; both direct costs (for example, medication, health care visits, and transportation) and indirect costs (for example, decreased productivity and home assistance) may increase with glaucoma progression. ${ }^{2}$

Although glaucomatous functional damage may be associated with multiple sources of neuronal insult, intraocular pressure (IOP) reduction is currently the only evidencebased treatment used for managing glaucoma with the aim of preventing, or at least slowing, the progression of visual field defects. ${ }^{3-6}$ The first approach for managing glaucoma generally relies on decreasing IOP through pharmacotherapy, which is commonly initiated through ophthalmic instillation of a single drug. ${ }^{7}$ Clinical studies have demonstrated that many patients require two or more drugs to achieve IOP decreases sufficient to slow or halt the progression of glaucoma..$^{5,8,9}$ Recently, there has been a trend 
toward increased use of multiple IOP-lowering antiglaucoma medications by Japanese patients, ${ }^{10,11}$ but complications such as risk of drug washout, additive exposure to preservatives, and decreased patient adherence to treatment may occur with use of multiple individual medications. ${ }^{12-15}$ Fixed-combination pharmacotherapies containing compounds with two or more hypotensive mechanisms of action in a single ophthalmic solution have been developed to avoid these complications, and they are expected to improve patient adherence. ${ }^{16,17}$

Timolol $0.5 \%$ (TIM) and brinzolamide 1\% (BRINZ) are generally well tolerated and effective at decreasing IOP. ${ }^{18-22}$ The fixed combination of BRINZ and TIM (BRINZ/TIM-FC) (Azarga $^{\circledR}$; Alcon Laboratories, Inc., Fort Worth, TX, USA) achieved clinically significant IOP reductions of greater magnitude than either TIM or BRINZ monotherapy in patients with open-angle glaucoma or ocular hypertension. ${ }^{23,24}$ Populationbased studies suggest that of the more than 60 million people worldwide diagnosed with glaucoma in 2010, 47\% were people of Asian descent, ${ }^{25}$ with an estimated prevalence of primary open-angle glaucoma of 3.9\% in Japanese patients. ${ }^{26}$ Although there are published reports of BRINZ/TIM-FC in the United States ${ }^{27}$ and in the European Union, ${ }^{8,28}$ there are no reports of clinical studies or clinical experiences of the efficacy and safety of BRINZ/TIM-FC in Japanese patients. There are, however, published reports of studies of fixed and unfixed dorzolamide 1\%/TIM combination therapy in Japanese patients. ${ }^{17,29}$ The objective of the current double-masked study was to evaluate the efficacy and safety of twice-daily BRINZ/ TIM-FC therapy compared with TIM monotherapy in Japanese patients with open-angle glaucoma or ocular hypertension.

\section{Patients and methods}

\section{Study design}

This was a randomized, double-masked, multicenter, 12-week clinical trial consisting of a 4-week observation period in which all patients received TIM, as well as an 8 -week treatment period in which patients received TIM or BRINZ/TIM-FC (Figure 1). At the initial visit, IOP was assessed, and patients were required to discontinue their currently used IOP-lowering drugs. At the conclusion of the initial visit, patients were instructed to instill 1 drop of TIM in each eye at 9 am $( \pm 30$ minutes $)$ and 9 pm ( \pm 30 minutes $)$ during the observation period (at least 4 weeks). At the second visit (week 0), patients were assessed for baseline IOP at 9 am and $11 \mathrm{am}$, and for whether or not they met the inclusion and exclusion criteria of the present study. Patients were randomized to BRINZ/TIM-FC or TIM monotherapy groups by a minimization method that adapted the randomization process by taking baseline IOP values into account.

For all sites, the research protocol was reviewed and approved by their respective institutional review board (Shin Akasaka Clinic, Tokyo, Japan; Hanna Hospital, Osaka, Japan; Sone Clinic, Tokyo, Japan; Mie University Hospital, Mie, Japan; and Osaka Pharmacology Clinical Research Hospital, Osaka, Japan) and was conducted according to the Declaration of Helsinki. Patients were included after approval from each institutional review board; study participants provided written informed consent before enrollment.

\section{Patients}

Participants in this study were Japanese patients aged 20 years or older who were diagnosed with open-angle glaucoma (primary open-angle glaucoma, exfoliation glaucoma, or pigmentary glaucoma) or ocular hypertension by the study investigators. The patients had a baseline IOP of $18-36 \mathrm{mmHg}$ in at least one eye, and an IOP of $36 \mathrm{mmHg}$ or lower in both eyes. Women who were pregnant, nursing, or planning to become pregnant during the study were excluded, as were patients currently or previously (within 30 days

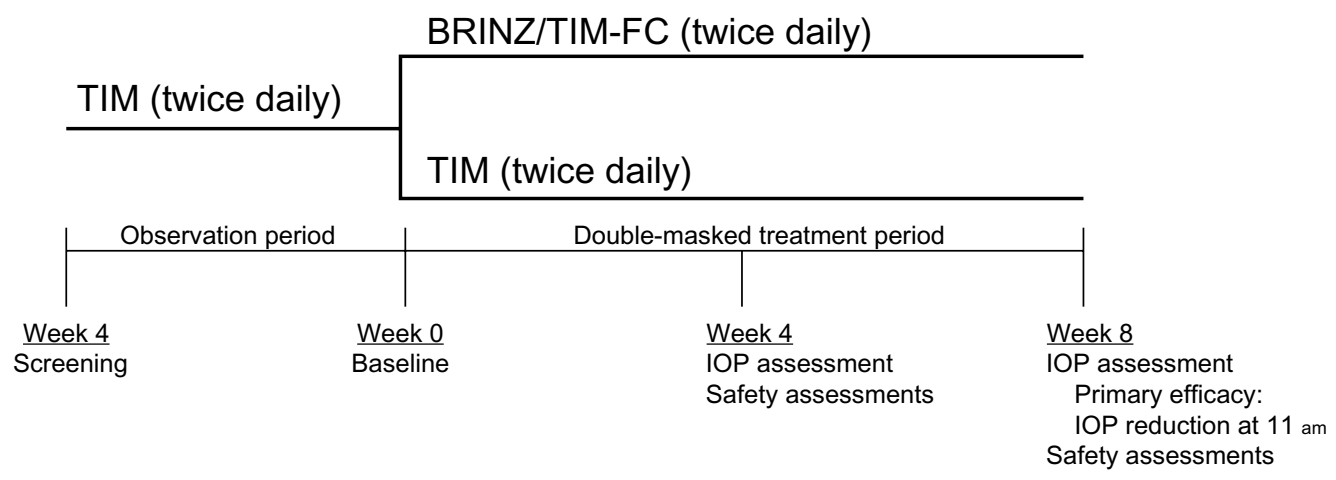

Figure I Study design.

Abbreviations: BRINZ/TIM-FC, brinzolamide 1\%/timolol 0.5\% fixed combination; TIM, timolol 0.5\%; IOP, intraocular pressure. 
of screening) participating in other studies. Also excluded were patients judged as ineligible for participation in the present study, as per the opinion of the investigators, as well as patients with the following conditions or medical histories: chronic or recurrent severe ocular inflammatory disease; current ocular infection; ocular trauma or intraocular surgery within 6 months of screening; any abnormality preventing reliable applanation tonometry; retinal disease; hypersensitivity to the study drugs; or use of confounding ophthalmic or systemic drugs.

\section{Treatment and masking}

Patients received either TIM ophthalmic solution or BRINZ/ TIM-FC ophthalmic suspension. Matched placebos for TIM and BRINZ/TIM-FC (both consisting of aqueous ophthalmic solutions containing no active ingredients) were provided in bottles that were identical to those of their matched study drug (ie, the double-dummy method) to ensure double-masking during the treatment phase. Patients were instructed to instill one drop of the active drug (BRINZ/TIM-FC or TIM) and one drop of the appropriate placebo (TIM placebo or BRINZ/ TIM-FC placebo) 5 minutes apart following the same twicedaily treatment regimen used during the observation period. At all study visits, instillation of 9 am doses was performed by a third party (an unmasked on-site pharmacist) and was not observed by masked investigators. The pharmacist who instilled study treatments/placebos did not disclose information regarding the nature of the study drugs to the patients, and the investigator and pharmacist instructed patients not to discuss the nature of study drugs used by the patients. Patients were requested to record their actual treatment administration between visits in journals, including the time of the 9 am instillation on visit days.

\section{IOP-lowering efficacy assessments}

IOP was assessed by Goldmann applanation tonometry once at the first visit, at 9 am (before drug instillation, 12 hours after administration of the previous evening dose; efficacy trough time point $)^{30,31}$ and at 11 am (2 hours postinstillation; efficacy peak time point for both BRINZ/TIM-FC and TIM) ${ }^{30-32}$ at the second (baseline) visit, 4-week treatment period visit, and 8-week treatment period visit. The primary efficacy endpoint was mean IOP reduction from baseline at $11 \mathrm{am}$ (peak) after 8 weeks of treatment with BRINZ/TIM-FC versus TIM monotherapy. IOP reduction at 9 am (trough) at week 8, mean IOP over time, and the percent IOP reduction from baseline served as secondary efficacy variables.

\section{Safety assessments}

Adverse events (AEs) were assessed at the baseline, week 4 , and week 8 visits, and classified using the Medical Dictionary for Regulatory Activities Japanese translation, version 14.1. ${ }^{33}$ Best-corrected visual acuity (decimal acuity scale), slit-lamp examination (cornea, eyelid/ conjunctiva, iris/anterior chamber, lens), blood pressure, and pulse rate were assessed at screening, baseline, and at treatment weeks 4 and 8. Fundoscopy (vitreous, retina/ macula/choroid, optic nerve, optic nerve cup-to-disc ratio), gonioscopy (anterior chamber angle grade), and laboratory tests (hematology, serum chemistry, qualitative urine test) were performed at screening, baseline, and week 8.

\section{Statistical analyses}

The least squares mean of IOP reduction from baseline in each group, and the difference between groups, were estimated by repeated-measures analysis of covariance. Baseline IOP values were used as the covariate. BRINZ/TIM-FC was considered superior to TIM if the difference in mean IOP reduction from baseline between groups at $11 \mathrm{am}$ at the 8-week treatment period visit (primary endpoint) was statistically significant, as verified by a $t$-test. No adjustment for a type 1 (alpha) error rate was performed for the primary efficacy analysis because it was based on a treatment difference of a single time point (11 am at week 8). The two-sided significance level was 0.05 . Other $P$-values were calculated for exploratory purposes.

Descriptive statistics were obtained for mean IOP and the degree of IOP reduction from baseline throughout the treatment period. Efficacy was assessed in the intent-totreat (ITT) population, which included patients who were administered the drug during the treatment period and for whom postadministration tests and observation data existed. AEs were evaluated in all patients administered the investigational drug during the treatment period. $P$-values of the treatment group differences for the baseline demographics and AEs were based on chi-square tests, Fisher's exact tests, or $t$-tests.

\section{Results}

This study was conducted from November 2010 to July 2011 at 31 study sites across Japan. Of the 321 patients screened in the observation period, 301 eligible patients (93.8\%; BRINZ/ TIM-FC, $\mathrm{n}=150$; TIM, $\mathrm{n}=151$; mean \pm standard deviation [SD] age, $61 \pm 13$ years) were enrolled in the treatment period and included in the ITT and safety populations. A total of 


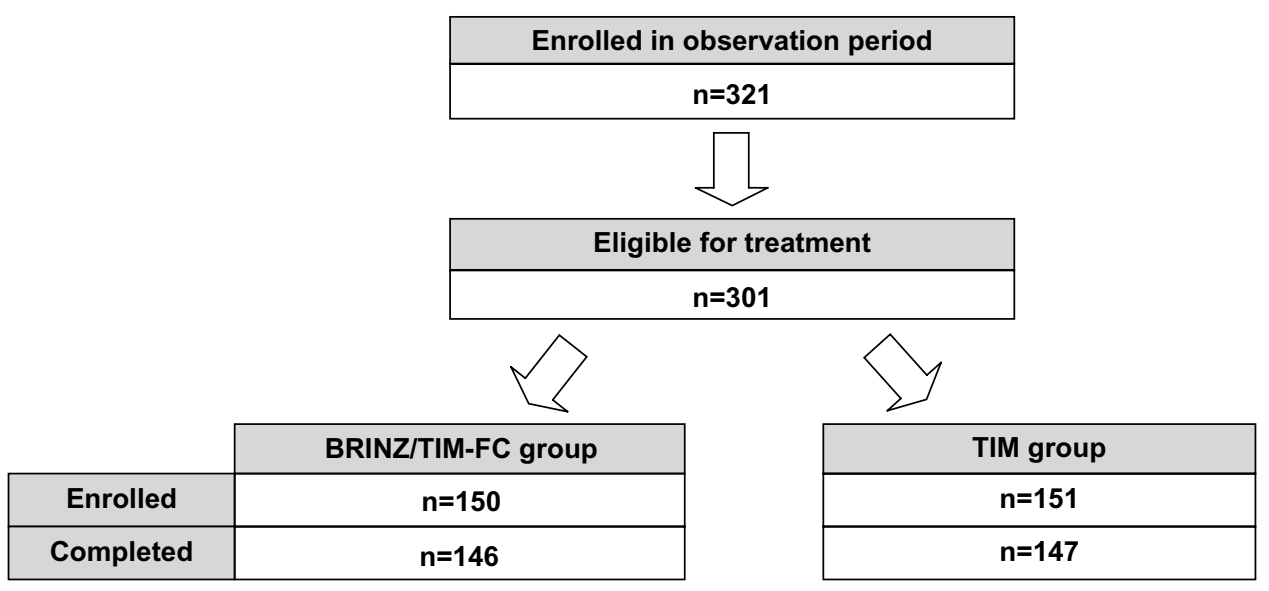

Figure 2 Patient disposition.

Note: Intent-to-treat and safety populations, $\mathrm{n}=30 \mathrm{I}$.

Abbreviations: BRINZ/TIM-FC, brinzolamide 1\%/timolol $0.5 \%$ fixed combination; TIM, timolol $0.5 \%$; $n$, number of patients.

293 patients (BRINZ/TIM-FC, n=146; TIM, n=147) completed the treatment period (Figure 2$)$. Eight patients $(2.7 \%$; BRINZ/TIM-FC, n=4; TIM, n=4) discontinued the study during the treatment period. Reasons for discontinuation were as follows: AEs (BRINZ/TIM-FC, $n=1$; TIM, $n=3$ ), patient requirement (BRINZ/TIM-FC, $\mathrm{n}=2$ ), and patient relocation (TIM, $\mathrm{n}=1$ ); one additional patient was discontinued because of insufficient IOP reduction with BRINZ/TIM-FC and was subsequently treated with a different IOP-lowering medication. No significant differences in sex, age, or diagnosis were observed between treatment groups (Table 1; $P \geq 0.5087)$. Most study participants (99.7\%) were diagnosed with either primary open-angle glaucoma (52.2\%) or ocular hypertension (47.5\%).
IOP analyses are presented only for the ITT population (primary analyses). No significant differences in the mean \pm SD baseline IOP were observed between treatment groups receiving BRINZ/TIM-FC (9 am, 20.8 $\pm 2.6 \mathrm{mmHg}$; $11 \mathrm{am}, 20.7 \pm 2.5 \mathrm{mmHg}$ ) or TIM (9 am, 20.8 $\pm 2.5 \mathrm{mmHg}$; $11 \mathrm{am}, 20.7 \pm 2.7 \mathrm{mmHg} ; P \geq 0.9767)$. Mean IOP values by treatment over time are presented in Figure 3. When mean IOP reductions with BRINZ/TIM-FC versus TIM were compared over time (Figure 4), the mean IOP reduction with BRINZ/ TIM-FC was significantly greater than with TIM at week 8 at $11 \mathrm{am}$ (primary endpoint, approximate peak time point for BRINZ/TIM-FC and TIM; $P<0.0001)$. Furthermore, mean IOP reductions with BRINZ/TIM-FC were greater than with TIM at week 8 at 9 am (trough time point, 12 hours after the

Table I Patient demographics and diagnosis

\begin{tabular}{|c|c|c|c|c|}
\hline & $\begin{array}{l}\text { BRINZ/TIM-FC } \\
(n=150)\end{array}$ & $\begin{array}{l}\text { TIM } \\
(n=\mid 51)\end{array}$ & $\begin{array}{l}\text { All patients } \\
(n=30 I)\end{array}$ & $P$-value (test)* \\
\hline Sex, n (\%) & & & & $0.856 \mathrm{I}$ (chi-square test) \\
\hline Male & $65(43.3)$ & $67(44.4)$ & 132 (43.9) & \\
\hline Female & $85(56.7)$ & $84(55.6)$ & $169(56.1)$ & \\
\hline Mean (SD) age, years & $61(13.7)$ & $62(12.5)$ & $61(13.1)$ & 0.5087 (t-test) \\
\hline \multicolumn{5}{|l|}{ Age group in years, $n(\%)$} \\
\hline$<65$ & $81(54.0)$ & $82(54.3)$ & $163(54.2)$ & 0.9577 (chi-square test)** \\
\hline$\geq 65$ & $69(46.0)$ & $69(45.7)$ & I $38(45.8)$ & \\
\hline$\geq 65$ to $<75$ & $42(28.0)$ & $46(30.5)$ & $88(29.2)$ & 0.8943 (Fisher's exact test)*** \\
\hline$\geq 75$ to $<85$ & $26(17.3)$ & $22(14.6)$ & $48(15.9)$ & \\
\hline$\geq 85$ to $<95$ & I $(0.7)$ & I (0.7) & $2(0.7)$ & \\
\hline Diagnosis, n (\%) & & & & I.0000 (Fisher's exact test) \\
\hline Primary open-angle glaucoma & $78(52.0)$ & $79(52.3)$ & $157(52.2)$ & \\
\hline Exfoliation glaucoma & $0(0.0)$ & I (0.7) & I $(0.3)$ & \\
\hline Pigmentary glaucoma & $0(0.0)$ & $0(0.0)$ & $0(0.0)$ & \\
\hline Ocular hypertension & $72(48.0)$ & $7 I(47.0)$ & I 43 (47.5) & \\
\hline
\end{tabular}

Notes: *BRINZ/TIM-FC versus TIM; *** $<5$ years versus $\geq 65$ years; $* * *<65$ years versus $\geq 65$ to $<75$ years versus $\geq 75$ years to $<85$ years versus $\geq 85$ years to $<95$ years. Abbreviations: BRINX/TIM-FC, brinzolamide 1\%/timolol 0.5\% fixed combination; n, number of patients; TIM, timolol 0.5\% monotherapy; SD, standard deviation. 


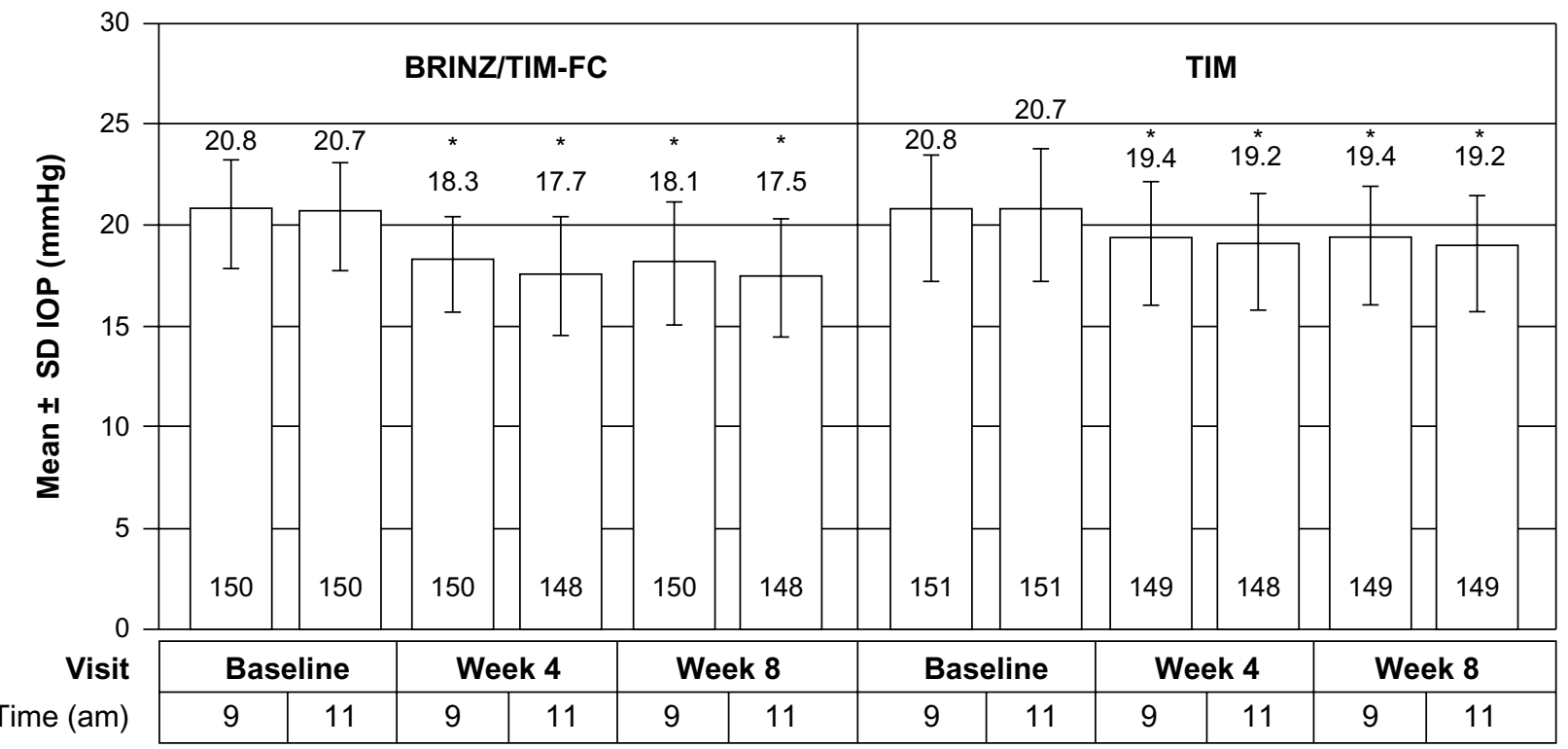

Figure 3 Mean \pm SD IOP over time (ITT).

Notes: Patient numbers for each group and time point are indicated in bars; IOP (descriptive mean) is indicated above the bars. $* P<0.05$ versus time-matched baseline; $P<0.05$ for BRINZ/TIM-FC versus TIM at all time points, repeated measures analysis of covariance of least squares means, adjusted with baseline IOP.

Abbreviations: BRINZ/TIM-FC, brinzolamide I\%/timolol 0.5\% fixed combination; SD, standard deviation; IOP, intraocular pressure; ITT, intent-to-treat population; TIM, timolol $0.5 \%$.

evening dose) and at week 4 at 9 am and 11 am (Figure 4). At week 8 , during the 11 am time point, and all other time points, significantly higher percentages of patients in the BRINZ/ TIM-FC group had greater reductions in IOP from baseline versus those in the TIM group (Figure 5). Mean IOP percent reductions from baseline with BRINZ/TIM-FC were significantly larger when compared with TIM monotherapy at all time points assessed $(P<0.05$; Figure 6$)$.
The incidence of AEs was $20 \%$ of patients in the BRINZ/ TIM-FC group $(n=30 / 150)$ and $19 \%$ in the TIM group ( $n=28 / 151)$; there was no significant difference between groups ( $P=0.7486$; chi-square test). Study discontinuations due to AEs were observed for four patients (BRINZ/ TIM-FC, $\mathrm{n}=1$ [eye irritation/abnormal feeling]; TIM, $\mathrm{n}=3$ [punctate keratitis, dry eye/eye pain, and allergic conjunctivitis/eyelid eczema; one patient each]). These events

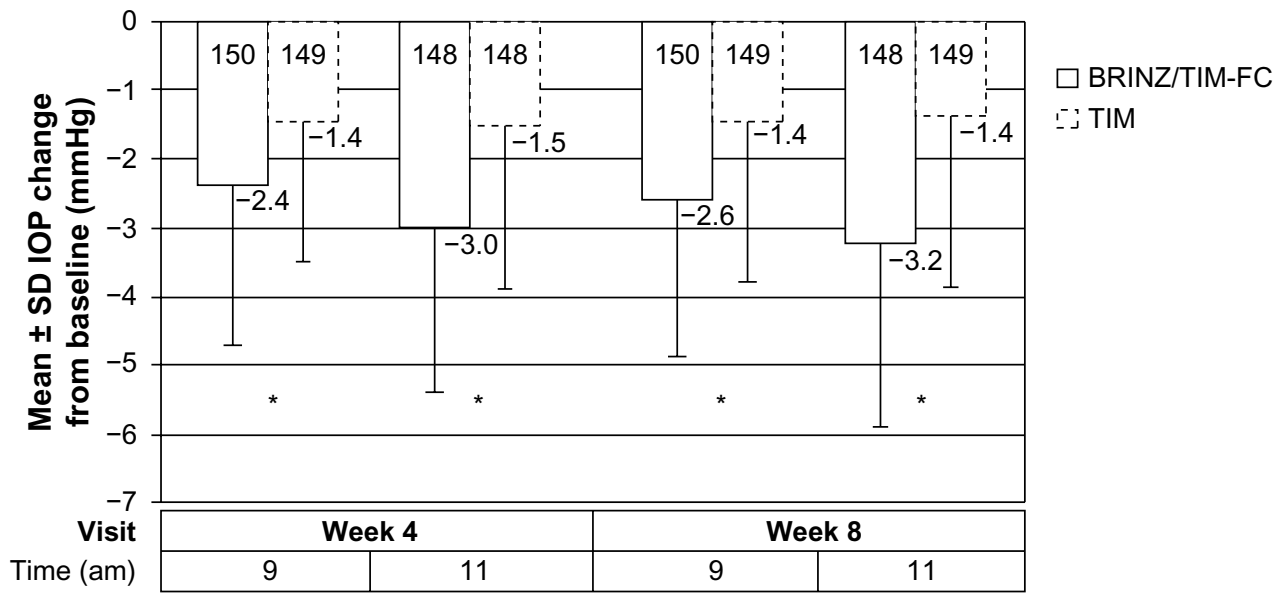

Figure 4 Mean \pm SD IOP reduction from baseline (ITT).

Notes: Patient numbers for each group and time point are indicated in bars; IOP (descriptive mean) is indicated the below bars. *P $<0.05$ for BRINZ/TIM-FC versus TIM, repeated measures analysis of covariance of least squares means, adjusted with baseline IOP.

Abbreviations: BRINZ/TIM-FC, brinzolamide I\%/timolol 0.5\% fixed combination; SD, standard deviation; IOP, intraocular pressure; ITT, intent-to-treat population; TIM, timolol $0.5 \%$. 


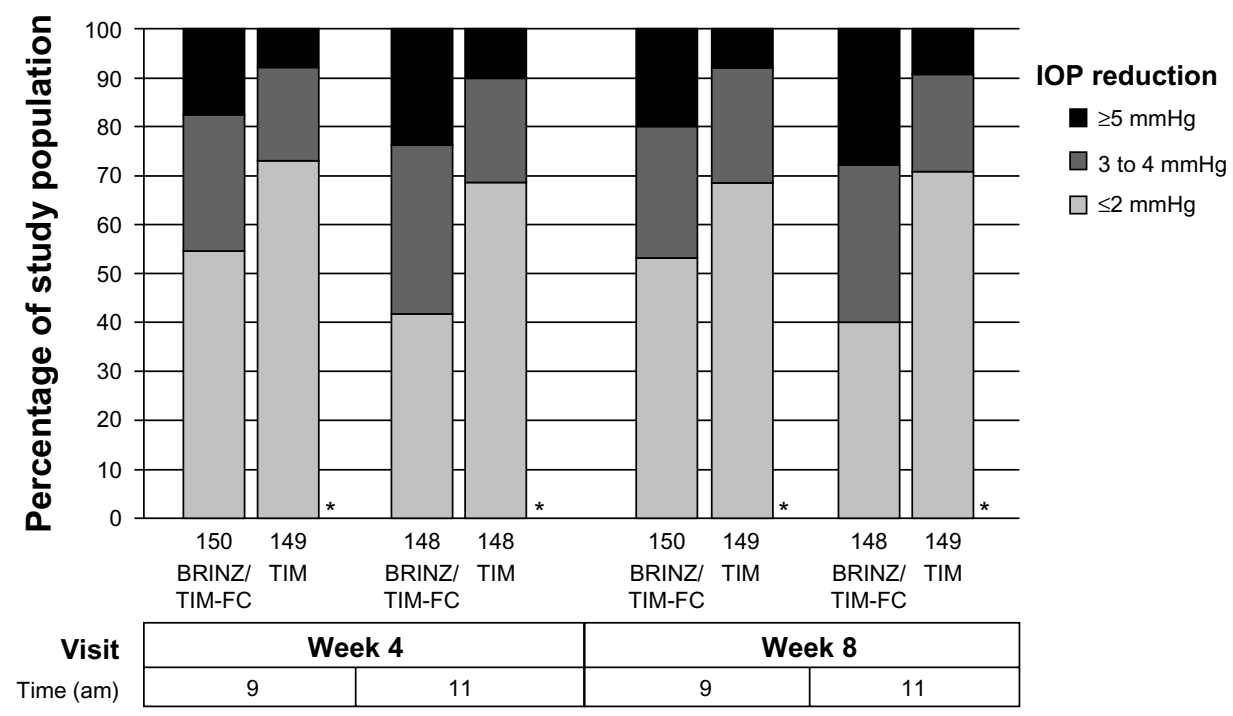

Figure 5 Rates of IOP reduction from baseline by magnitude of change (ITT).

Notes: Group sizes are indicated beneath the bars. Percentages of patients with IOP reduction from baseline of $2 \mathrm{mmHg}$ or less, $3-4 \mathrm{mmHg}$, and $5 \mathrm{mmHg}$ or more (last observation carried forward) are shown. $* P<0.05$ for BRINZ/TIM-FC versus TIM, Wilcoxon rank-sum test.

Abbreviations: BRINZ/TIM-FC, brinzolamide I\%/timolol $0.5 \%$ fixed combination; IOP, intraocular pressure; ITT, intent-to-treat population; TIM, timolol 0.5\%.

resolved with or without treatment. No serious AEs were reported.

All side effects (ie, AEs for which a causal relationship with treatment could not be ruled out) reported in either treatment group are presented in Table 2. A total of 18 of 301 patients ( $6 \%$ overall; BRINZ/TIM-FC, $\mathrm{n}=13 / 150$ [9\%]; TIM, $\mathrm{n}=5 / 151[3 \%]$ ) experienced side effects (treatment group difference, $P=0.0501$; chi-square test). Side effects occurring in more than one patient in the BRINZ/TIM-FC treatment group included eye irritation ( $\mathrm{n}=7 / 150[5 \%])$, blurred vision $(n=2 / 150[1 \%])$, and conjunctival hyperemia $(n=2 / 150[1 \%])$, and in the TIM treatment group included punctate keratitis and conjunctival hyperemia ( $\mathrm{n}=2 / 151,[1 \%]$ each). Eye irritation was observed only in the BRINZ/TIM-FC group; these events resolved without treatment and were of mild intensity, except in one patient who reported moderate eye irritation and discontinued the study.

No safety issues were observed with either BRINZ/ TIM-FC or TIM according to ocular safety assessments. No significant changes from baseline in mean pulse rate were observed with either treatment (Table 3). Small-magnitude decreases from baseline in terms of mean systolic and diastolic blood pressure were observed in the BRINZ/TIM-FC and TIM groups; no significant differences were observed

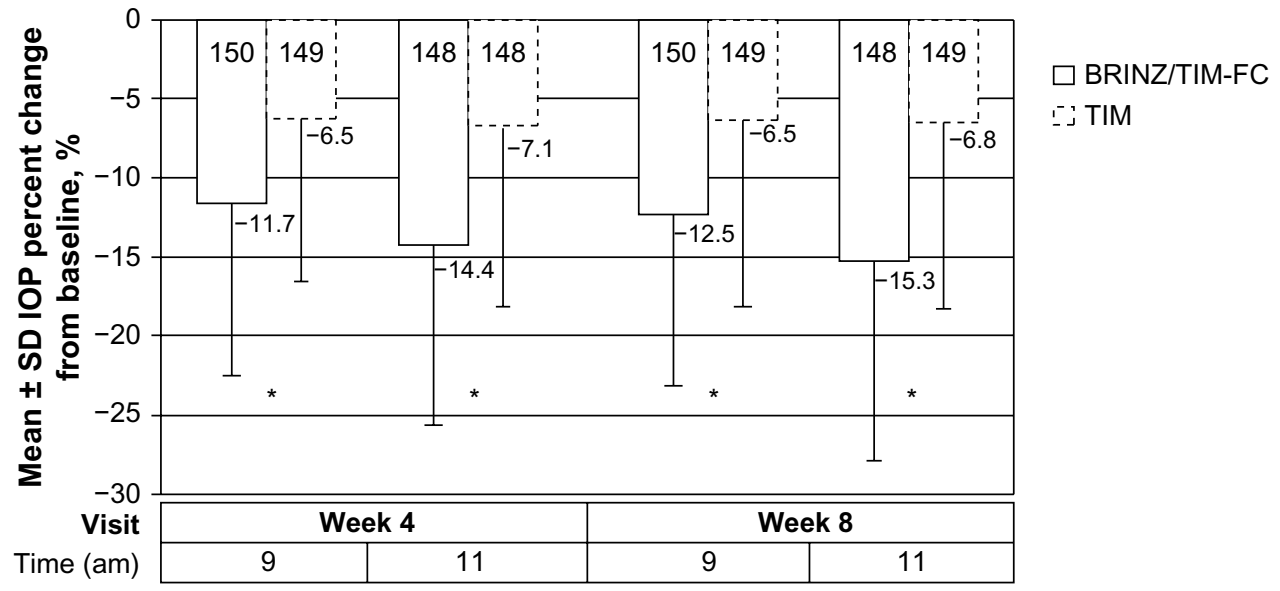

Figure 6 IOP percent reduction from baseline (ITT).

Notes: Patient numbers for each group and time point are indicated in bars; IOP (descriptive mean) is indicated below. $* P<0.05$ for BRINZ/TIM-FC versus TIM, repeated measures analysis of covariance of least squares means, adjusted with baseline IOP.

Abbreviations: BRINZ//TIM-FC, brinzolamide 1\%/timolol 0.5\% fixed combination; SD, standard deviation; IOP, intraocular pressure; ITT, intent-to-treat population; TIM, timolol $0.5 \%$. 
Table 2 Incidence of AEs by treatment group*

\begin{tabular}{|c|c|c|}
\hline \multirow[t]{2}{*}{ Coded AE** } & \multicolumn{2}{|c|}{ AEs related to therapy } \\
\hline & $\begin{array}{l}\text { BRINZ/TIM-FC } \\
(n=150)\end{array}$ & $\begin{array}{l}\text { TIM } \\
(n=\mid 5 I)\end{array}$ \\
\hline Total AEs, n (\%)*** & $30(20)$ & $28(19)$ \\
\hline Total AEs related to therapy, $\mathrm{n}(\%)^{\S}$ & $13(9)$ & $5(3)$ \\
\hline \multicolumn{3}{|l|}{ Eye disorders } \\
\hline Eye irritation & $7(5)$ & $0(0)$ \\
\hline Blurred vision & $2(1)$ & $I(1)$ \\
\hline Conjunctival hyperemia & $2(1)$ & $2(1)$ \\
\hline Punctate keratitis & $I(1)$ & $2(1)$ \\
\hline Eye pain & $\mathrm{I}(\mathrm{I})$ & $0(0)$ \\
\hline Eczema eyelids & $0(0)$ & $\mathrm{I}(\mathrm{I})$ \\
\hline \multicolumn{3}{|l|}{ Nervous system disorders } \\
\hline Dysgeusia & $I(1)$ & $0(0)$ \\
\hline \multicolumn{3}{|l|}{$\begin{array}{l}\text { General disorders and administration } \\
\text { site conditions }\end{array}$} \\
\hline Feeling abnormal & $\mathrm{I}(\mathrm{I})$ & $0(0)$ \\
\hline \multicolumn{3}{|l|}{ Investigations } \\
\hline Blood lactate dehydrogenase increased & $\mathrm{I}(\mathrm{I})$ & $0(0)$ \\
\hline Blood potassium increased & $\mathrm{I}(\mathrm{I})$ & $0(0)$ \\
\hline
\end{tabular}

Notes: *Total evaluable patients (safety population), $\mathrm{n}=30 \mathrm{I}$. **Terminology from: Medical Dictionary for Regulatory Activities Japanese translation, version 14.1. ${ }^{33}$ ***Treatment group difference for total $A E s, P=0.7848$, chi-square test. ${ }^{\S}$ Treatment group difference for treatment-related $A E s, P=0.050 \mathrm{I}$, chi-square test.

Abbreviations: AE, adverse event; BRINZ/TIM-FC, brinzolamide I\%/timolol $0.5 \%$ fixed combination; $n$, number of patients reporting AEs/total group size; TIM, timolol $0.5 \%$ monotherapy.

for pulse rate or blood pressure changes from baseline with BRINZ/TIM-FC versus TIM (Table 3). Increased levels of blood lactate dehydrogenase and potassium were each observed in $1 / 150$ patients receiving BRINZ/TIM-FC $(0.7 \%)$ and were reported as AEs related to treatment. Both AEs were mild in severity and improved or resolved without treatment.

\section{Discussion}

The objective of the current study was to evaluate the efficacy and safety of BRINZ/TIM-FC compared with TIM monotherapy in Japanese patients with open-angle glaucoma or ocular hypertension. BRINZ/TIM-FC therapy was associated with significantly greater reductions in IOP compared with TIM monotherapy at the estimated peak (primary endpoint) and trough (secondary endpoint) time points, and it was well tolerated in this study population. Overall, the efficacy and safety profiles of BRINZ/TIM-FC observed in the present study were consistent with those reported in previous investigations of BRINZ/TIM-FC, BRINZ monotherapy, or concomitant BRINZ and TIM therapy in other patient populations. ${ }^{8,21,23,24,27,34,35}$ The double-masked, randomized design of this study increases the confidence in the findings presented here.
In the current study, both BRINZ/TIM-FC and TIM significantly reduced IOP from baseline; IOP reductions with BRINZ/TIM-FC therapy were significantly greater than with TIM at week 8 at 11 am (primary endpoint; estimated peak time point for BRINZ/TIM-FC and TIM, 2 hours after the morning dose). Furthermore, mean IOP reductions with BRINZ/TIM-FC were greater than with TIM at week 8 at 9 am (secondary endpoint, estimated trough time point, 12 hours after the evening dose) and at week 4 at 9 am and $11 \mathrm{am}$. At the $11 \mathrm{am}$ assessment of the week 8 visit (2 hours postinstillation; peak time point for BRINZ/TIM-FC and TIM), the mean IOP reduction in patients receiving BRINZ/ TIM-FC $(-3.2 \mathrm{mmHg})$ was twice that of patients receiving TIM $(-1.4 \mathrm{mmHg})$. After $4-8$ weeks of treatment with BRINZ/TIM-FC, the mean IOP percent reduction from baseline ranged from $11.7 \%-15.3 \%$, compared with the $6.5 \%-7.1 \%$ reduction noted with TIM. Furthermore, IOP was reduced by $3 \mathrm{mmHg}$ or more in approximately half of patients who received BRINZ/TIM-FC, compared with one-third of patients who received TIM, resulting in significantly higher rates of IOP reduction in the BRINZ/TIM-FC treatment group ( $P<0.05$; Wilcoxon rank-sum test). After a 4-week observation period of TIM monotherapy, BRINZ/TIM-FC led to additional IOP reductions of approximately $5 \%-8 \%$ beyond those achieved with continued TIM treatment. The mean IOP reductions from baseline at weeks 4 and 8 with continued TIM treatment were statistically significant, but they were $1.5 \mathrm{mmHg}$ or less; these reductions were considered to be within the range of normal variation and therefore not clinically relevant. ${ }^{36,37}$

There have been several studies on BRINZ/TIM-FC reported prior to the present study. One study of more than 10,000 German patients demonstrated the IOP-lowering efficacy of BRINZ/TIM-FC, with IOP reductions from baseline of nearly $4 \mathrm{mmHg}$; however, this was an open-label clinical trial in a study population that included previously untreated patients. ${ }^{38} \mathrm{~A}$ double-masked study compared the IOP-lowering efficacy of TIM monotherapy versus BRINZ/ TIM-FC in patients from the United States who were previously untreated or who had undergone a sufficient washout of any prior ocular hypotensives before initiation of the study. ${ }^{23}$ Results of that study showed significantly greater IOP-lowering efficacy with BRINZ/TIM-FC compared with TIM monotherapy (mean IOP difference for BRINZ/TIMFC versus TIM, $1.3-1.8 \mathrm{mmHg}$ ) and also demonstrated the clinical utility of BRINZ/TIM-FC for patients with glaucoma or ocular hypertension. However, these studies evaluated BRINZ/TIM-FC in patients from Europe and North America, 
Table 3 Pulse rate and blood pressure

\begin{tabular}{|c|c|c|c|c|c|c|}
\hline & \multirow{2}{*}{\multicolumn{2}{|c|}{$\begin{array}{l}\text { Baseline } \\
\text { Week } 0\end{array}$}} & \multicolumn{4}{|c|}{ Change from baseline } \\
\hline & & & \multicolumn{2}{|l|}{ Week 4} & \multicolumn{2}{|l|}{ Week 8} \\
\hline & 9 am & II am & 9 am & II am & 9 am & II am \\
\hline \multicolumn{7}{|c|}{ Pulse rate, BPM } \\
\hline \multicolumn{7}{|c|}{ BRINZ/TIM-FC } \\
\hline Mean (SD) & $69.5(9.2)$ & $63.4(9.0)$ & $-0.9(6.9)$ & $0.8(7.3)$ & $0.2(7.6)$ & $0.4(7.8)$ \\
\hline $\mathrm{N}$ & 150 & 150 & 150 & 148 & 147 & 146 \\
\hline$P$-value* & - & - & 0.1627 & 0.2018 & $0.706 I$ & 0.4671 \\
\hline \multicolumn{7}{|l|}{ TIM } \\
\hline Mean (SD) & $70.5(11.0)$ & $64.2(8.7)$ & $0.5(8.6)$ & $0.6(6.5)$ & $-0.1(8.2)$ & $-0.5(6.4)$ \\
\hline $\mathrm{N}$ & $|5|$ & 151 & 149 & 148 & 147 & 148 \\
\hline$P$-value* & - & - & 0.4074 & 0.2594 & 0.9040 & $0.5|4|$ \\
\hline \multicolumn{7}{|c|}{ Systolic blood pressure, $\mathrm{mmHg}$} \\
\hline \multicolumn{7}{|c|}{ BRINZ/TIM-FC } \\
\hline Mean (SD) & |34.I (21.4) & $132.0(19.3)$ & $-1.4(13.9)$ & $-0.7(\mid 3.0)$ & $-2.3(13.2)$ & $-2.4(12.5)$ \\
\hline $\mathrm{N}$ & 150 & 150 & 150 & 148 & 147 & 146 \\
\hline$P$-value* & - & - & 0.2018 & 0.5197 & 0.0280 & 0.0300 \\
\hline \multicolumn{7}{|l|}{ TIM } \\
\hline Mean (SD) & | $34.9(21.8)$ & I30.4 (21.6) & $-3.3(10.7)$ & $-I . I(13.4)$ & $-3.2(13.7)$ & $-1.6(13.7)$ \\
\hline $\mathrm{N}$ & $15 \mid$ & $15 \mid$ & 149 & 148 & 147 & 148 \\
\hline$P$-value* & - & - & 0.0027 & 0.3189 & 0.0040 & 0.1455 \\
\hline \multicolumn{7}{|c|}{ Diastolic blood pressure, $\mathrm{mmHg}$} \\
\hline \multicolumn{7}{|c|}{ BRINZ/TIM-FC } \\
\hline Mean (SD) & $79.5(12.5)$ & $78.2(12.1)$ & $-0.8(9.1)$ & $0.1(8.6)$ & $-1.7(8.4)$ & $-0.9(9.0)$ \\
\hline $\mathrm{N}$ & 150 & 150 & 150 & 148 & 147 & 146 \\
\hline$P$-value* & - & - & 0.2678 & 0.9302 & 0.0223 & 0.2680 \\
\hline \multicolumn{7}{|l|}{ TIM } \\
\hline Mean (SD) & $79.7(12.8)$ & $78.7(12.9)$ & $-1.3(8.2)$ & $-0.8(9.1)$ & $-1.5(9.8)$ & $-1.9(9.7)$ \\
\hline $\mathrm{N}$ & $|5|$ & $|5|$ & 149 & 148 & 147 & 148 \\
\hline$P$-value* & - & - & 0.0755 & 0.2939 & 0.0401 & 0.0098 \\
\hline
\end{tabular}

Notes: $* P$-values were provided by $t$-test from repeated measures analysis of variance. For treatment comparisons, $P>0.05$ at all time points.

Abbreviations: BPM, beats per minute; BRINZ/TIM-FC, brinzolamide I\%/timolol $0.5 \%$ fixed combination; SD, standard deviation; N, number of patients; TIM, timolol $0.5 \%$.

and their findings may not be directly applicable to patients of Asian descent, including the Japanese patient population.

In view of these previous studies, we investigated the ocular hypotensive effect of BRINZ/TIM-FC versus TIM monotherapy, after a 4-week run-in period of TIM monotherapy, in Japanese patients using a double-masked study design. In principle, topical glaucoma therapies should be added one at a time, as needed, to achieve and maintain target IOP. ${ }^{7}$ The design of the current study reflects the use of fixed-combination drugs in the clinical practice setting because BRINZ/TIM-FC treatment was initiated after patients had been treated with TIM monotherapy without a washout period between the run-in (TIM) period and the observational (BRINZ/TIM-FC or TIM) period. Furthermore, the IOP reduction from baseline with twice-daily BRINZ/ TIM-FC at week 8 ( 2 hours postinstillation) reported in the present study is comparable to the IOP reduction reported with thrice-daily concomitant therapy with BRINZ and TIM after 2 months of treatment ( 2 hours postinstillation) in patients in the United States; ${ }^{24}$ thus, BRINZ/TIM-FC demonstrated significantly greater IOP reductions compared with TIM monotherapy at approximate peak and trough time points. These results suggest that BRINZ/TIM-FC may be clinically effective as a fixed-combination drug in Japanese patients.

Our results showing that both BRINZ/TIM-FC and TIM were associated with significant reductions in IOP from baseline are similar to those observed in a comparison of a fixed combination of the carbonic anhydrase inhibitor, dorzolamide 1\%, and TIM (Cosopt ${ }^{\circledR}$; Merck and Co., Inc., Whitehouse Station, NJ, USA) or TIM monotherapy that was conducted in Japan. ${ }^{39}$ In other countries, Cosopt is formulated with $2 \%$ dorzolamide, whereas in Japan, Cosopt contains 1\% dorzolamide. A study of the $1 \%$ dorzolamide formulation of Cosopt was conducted in a similar patient population (ie, Japanese patients with glaucoma or ocular hypertension) and with a similar study design and duration. ${ }^{39}$ Although a direct comparison of the efficacy of BRINZ/TIM-FC versus Cosopt 
( $1 \%$ dorzolamide/TIM) in Japanese patients has not been performed, it is noteworthy that the IOP reductions at peak ( 2 hours postinstillation) and trough ( 12 hours postinstillation) after 8 weeks of treatment with BRINZ/TIM-FC in the present study were $-3.2 \mathrm{mmHg}$ and $-2.7 \mathrm{mmHg}$, respectively, whereas reductions of $-2.54 \mathrm{mmHg}$ and $-1.89 \mathrm{mmHg}$ were reported after 8 weeks of treatment with Cosopt at 2 hours and 12 hours postinstillation, respectively. ${ }^{39}$ Furthermore, a comparative, multinational trial of BRINZ/TIM-FC versus Cosopt ( $2 \%$ dorzolamide/TIM) previously demonstrated that the IOP-lowering efficacy of BRINZ/TIM-FC was thought to be noninferior to that of Cosopt. ${ }^{28}$

The overall incidence of AEs in this study was 19\%, with $19 \%$ of patients receiving TIM and $20 \%$ of patients receiving BRINZ/TIM-FC reporting AEs. No serious AEs were reported with BRINZ/TIM-FC or TIM in this study. Most AEs were mild or moderate, and most resolved without treatment. Six events of mild eye irritation and one event of mild dysgeusia, which was considered to be treatment-related, were reported by patients in the BRINZ/ TIM-FC treatment group, but these events resolved without treatment. One patient treated with BRINZ/TIM-FC who discontinued from the study reported moderate eye irritation that resolved without treatment; all other reported cases of eye irritation were mild. The incidence of treatment-related blurred vision with BRINZ/TIM-FC was $1 \%$. This incidence is lower than reported elsewhere, ${ }^{27}$ possibly because patients were made aware during the informed consent process that blurred vision may occur with BRINZ/TIM-FC, and wiping eyelids after instillation is thought to reduce the incidence of blurred vision. ${ }^{40}$

Treatment-related eye irritation was reported more frequently in patients receiving BRINZ/TIM-FC (5\%) than in patients receiving TIM (0\%). Compared with BRINZ or BRINZ/TIM-FC, ocular instillation of dorzolamide or Cosopt ( $2 \%$ dorzolamide/TIM) is more commonly associated with ocular discomfort, including burning and stinging, ${ }^{8,27,35,41}$ and clinical preference studies have shown that BRINZ/ TIM-FC was favored over Cosopt by a majority of patients with a treatment preference. ${ }^{27,35,38}$ These factors may lead to better patient adherence to treatment with BRINZ/TIM-FC, particularly for the many Japanese patients with dry eye who might have increased sensitivity to ocular discomfort (for example, stinging, burning); however, no direct comparisons of BRINZ/TIM-FC and Cosopt in Japanese patients have been reported. Changes in other safety assessments, including hematology, pulse rate, and blood pressure, were either not statistically significant or of small magnitude with both
BRINZ/TIM-FC and TIM. Significant decreases in systolic and diastolic blood pressure were evident in the BRINZ/ TIM-FC and TIM treatment groups at one or more assessments at weeks 4 and 8; there were no significant differences in blood pressure measurements between the groups at any time point. However, the observed decreases in blood pressure were of small magnitude, were not considered to be clinically relevant, and were not associated with any AEs.

One limitation of this study is that central corneal thickness, which can influence IOP, was not assessed. Another potential limitation is that the study population lacked any cases of normal tension or angle-closure glaucoma, both of which are common in people of Japanese descent; ${ }^{25,42}$ however, the results described here provide the first evidence of the IOP-lowering efficacy of BRINZ/TIM-FC in Japanese patients. The results also demonstrate that BRINZ/TIM-FC had superior IOP-lowering efficacy compared with TIM at peak and trough time points in Japanese patients with openangle glaucoma and elevated IOP or ocular hypertension, and was not associated with serious AEs or side effects. The efficacy and safety described in the current study are comparable to the efficacy and safety reported with BRINZ/TIM-FC in Europe and North America. These findings indicate that BRINZ/TIM-FC may be useful for clinical use in Japan.

\section{Acknowledgments}

The authors wish to thank the following participating investigators: Mikki Arai (Arai Eye Clinic, Fukuoka, Japan); Yuko Asai (Iida Hospital, Nagano, Japan); Kazuko Hijikuro (Nagata Eye Clinic, Hyogo, Japan); Kaori Hirai (International Goodwill Hospital, Kanagawa, Japan); Yoshinori Iwama (Iwama Eye Clinic, Mie, Japan); Hitoshi Kato (Kato Eye Clinic, Fukuoka, Japan); Takuji Kato (Kato Eye Clinic, Tokyo, Japan); Hidehito Kawabata (Kusakari Eye Clinic, Chiba, Japan); Sukehito Kure (Shisui Ophthalmologic Hospital, Chiba, Japan); Tsutomu Manabe (Manabe Clinic, Tokyo, Japan); Atsuko Matsuhisa (Sakura Ophthalmologic Clinic, Shizuoka, Japan); Sakae Matsuzaki (Seijo Clinic, Tokyo, Japan); Masayoshi Migita (Migita Eye Institute, Oita, Japan); Masatada Mitsuhashi (Mitsuhashi Eye Clinic, Chiba, Japan); Tomoyuki Muramatsu (Muramatsu Eye Clinic, Shizuoka, Japan); Hisayoshi Nanba (Nanba Eye Clinic, Shizuoka, Japan); Masahiko Nohara (Maruko Central General Hospital, Nagano, Japan); Shingo Onoe (Onoe Eye Clinic, Osaka, Japan); Yoshinari Sadamatsu (Sadamatsu Eye Clinic, Saitama, Japan); Isao Sato (Wakabadai Eye Clinic, Tokyo, Japan); Masaki Sato (Mito Kyodo General Hospital, Ibaraki, Japan); Tadayo Sato (Kusakari Eye Clinic, Chiba, 
Japan); Kiyoshi Shimizu (Kawaguchi Aozora Eye Clinic, Saitama, Japan); Keiji Sugasawa (Sugasawa Eye Clinic, Osaka, Japan); Miyuki Sugita (Maita Eye Clinic, Kanagawa, Japan); Torao Sugiura (Sugiura Eye Clinic, Osaka, Japan); Masao Takase (Takase Tairamachi Eye Clinic, Tokyo, Japan); Yuzuru Yoshimura (Yoshimura Eye and Internal Medical Clinic, Shizuoka, Japan).

This study was funded by Alcon Japan, Ltd. (Tokyo, Japan). Medical writing assistance was provided by Heather Starkey, PhD, of Complete Healthcare Communications, Inc. (Chadds Ford, PA, USA), and was funded by Alcon Laboratories, Inc.

\section{Author contributions}

All authors contributed to the study and to the manuscript preparation, and they reviewed and approved the manuscript.

\section{Disclosure}

Alcon Japan, Ltd. (Tokyo, Japan) and Alcon Laboratories, Inc. (Fort Worth, TX, USA) designed and conducted the study, and performed data collection, management, and analysis of data, and collaborated in manuscript writing. Dr Yoshikawa received compensation from Alcon for his work drafting and reviewing the manuscript; Dr Yoshikawa and Dr Kozaki have served as speakers for Alcon Japan, Ltd. Dr Maeda has no financial interests to disclose. The authors report no other conflicts of interest in this work.

\section{References}

1. van Gestel A, Webers CA, Beckers HJ, Peeters A, Severens JL, Schouten JS. Ocular hypertension and the risk of blindness. J Glaucoma. Epub March 20, 2013.

2. Varma R, Lee PP, Goldberg I, Kotak S. An assessment of the health and economic burdens of glaucoma. Am J Ophthalmol. 2011;152(4): 515-522.

3. Beckers HJ, Schouten JS, Webers CA. Role of fixed-combination brinzolamide $1 \% /$ timolol $0.5 \%$ in the treatment of elevated intraocular pressure in open-angle glaucoma and ocular hypertension. Clin Ophthalmol. 2009;3:593-599.

4. Webers CA, Beckers HJ, Nuijts RM, Schouten JS. Pharmacological management of primary open-angle glaucoma: second-line options and beyond. Drugs Aging. 2008;25(9):729-759.

5. Kass MA, Heuer DK, Higginbotham EJ, et al. The Ocular Hypertension Treatment Study: a randomized trial determines that topical ocular hypotensive medication delays or prevents the onset of primary openangle glaucoma. Arch Ophthalmol. 2002;120(6):701-713; discussion 829-830.

6. The Advanced Glaucoma Intervention Study (AGIS): 7. The relationship between control of intraocular pressure and visual field deterioration. The AGIS Investigators. Am J Ophthalmol. 2000;130(4): 429-440.

7. [The Japan Glaucoma Society Guidelines for Glaucoma (3rd Edition)]. Nihon Ganka Gakkai Zasshi. 2012;116(1):3-46. Japanese.
8. Auger GA, Raynor M, Longstaff S. Patient perspectives when switching from Cosopt $\left({ }^{\circledR}\right)$ (dorzolamide-timolol) to Azarga ${ }^{\mathrm{TM}}$ (brinzolamidetimolol) for glaucoma requiring multiple drug therapy. Clin Ophthalmol. 2012;6:2059-2062.

9. McCarty CA, Mukesh BN, Kitchner TE, et al. Intraocular pressure response to medication in a clinical setting: the Marshfield Clinic Personalized Medicine Research Project. J Glaucoma. 2008;17(5): 372-377.

10. Kashiwagi K. Changes in trend of newly prescribed anti-glaucoma medications in recent nine years in a Japanese local community. Open Ophthalmol J. 2010;4:7-11.

11. Kokuzawa S, Kondo Y, Yamamoto T. [Current status of medication for glaucoma in a university hospital]. Rinsho Ganka. 2006;60(9):16791684. Japanese.

12. Djafari F, Lesk MR, Harasymowycz PJ, Desjardins D, Lachaine J. Determinants of adherence to glaucoma medical therapy in a long-term patient population. J Glaucoma. 2009;18(3):238-243.

13. Sleath B, Robin AL, Covert D, Byrd JE, Tudor G, Svarstad B. Patientreported behavior and problems in using glaucoma medications. Ophthalmology. 2006;113(3):431-436.

14. Chrai SS, Makoid MC, Eriksen SP, Robinson JR. Drop size and initial dosing frequency problems of topically applied ophthalmic drugs. J Pharm Sci. 1974;63(3):333-338.

15. Pisella PJ, Pouliquen P, Baudouin C. Prevalence of ocular symptoms and signs with preserved and preservative free glaucoma medication. Br J Ophthalmol. 2002;86(4):418-423.

16. Higginbotham EJ. Considerations in glaucoma therapy: fixed combinations versus their component medications. Clin Ophthalmol. 2010;4:1-9.

17. Inoue K, Shiokawa M, Sugahara M, Wakakura M, Soeda S, Tomita G. Three-month evaluation of dorzolamide hydrochloride/timolol maleate fixed-combination eye drops versus the separate use of both drugs. Jpn J Ophthalmol. 2012;56(6):559-563.

18. Shedden A, Laurence J, Tipping R. Timoptic-XE 0.5\% Study Group. Efficacy and tolerability of timolol maleate ophthalmic gel-forming solution versus timolol ophthalmic solution in adults with open-angle glaucoma or ocular hypertension: a six-month, double-masked, multicenter study. Clin Ther. 2001;23(3):440-450.

19. Watson PG, Barnett MF, Parker V, Haybittle J. A 7 year prospective comparative study of three topical beta blockers in the management of primary open angle glaucoma. Br J Ophthalmol. 2001;85(8): 962-968.

20. Schenker H, Maloney S, Liss C, Gormley G, Hartenbaum D. Patient preference, efficacy, and compliance with timolol maleate ophthalmic gel-forming solution versus timolol maleate ophthalmic solution in patients with ocular hypertension or open-angle glaucoma. Clin Ther. 1999;21(1):138-147.

21. Sall K. The efficacy and safety of brinzolamide $1 \%$ ophthalmic suspension (Azopt) as a primary therapy in patients with open-angle glaucoma or ocular hypterension. Brinzolamide Primary Therapy Study Group. Surv Ophthalmol. 2000;44 Suppl 2:S155-S162.

22. Silver LH. Dose-response evaluation of the ocular hypotensive effect of brinzolamide ophthalmic suspension (Azopt). Brinzolamide Dose-Response Study Group. Surv Ophthalmol. 2000;44 Suppl 2: S147-S153.

23. Kaback M, Scoper SV, Arzeno G, et al. Brinzolamide 1\%/Timolol 0.5\% Study Group. Intraocular pressure-lowering efficacy of brinzolamide $1 \% /$ timolol $0.5 \%$ fixed combination compared with brinzolamide $1 \%$ and timolol 0.5\%. Ophthalmology. 2008;115(10):1728-1734, 1734. e1-2.

24. Shin D. Adjunctive therapy with brinzolamide $1 \%$ ophthalmic suspension (Azopt) in patients with open-angle glaucoma or ocular hypertension maintained on timolol therapy. Surv Ophthalmol. 2000;44 Suppl 2: S163-S168.

25. Quigley HA, Broman AT. The number of people with glaucoma worldwide in 2010 and 2020. Br J Ophthalmol. 2006;90(3):262-267. 
26. Iwase A, SuzukiY, Araie M, et al. Tajimi Study Group, Japan Glaucoma Society. The prevalence of primary open-angle glaucoma in Japanese: the Tajimi Study. Ophthalmology. 2004;111(9):1641-1648.

27. Mundorf TK, Rauchman SH, Williams RD, Notivol R. Brinzolamide/ Timolol Preference Study Group. A patient preference comparison of Azarga (brinzolamide/timolol fixed combination) vs Cosopt (dorzolamide/timolol fixed combination) in patients with open-angle glaucoma or ocular hypertension. Clin Ophthalmol. 2008;2(3): 623-628.

28. Manni G, Denis P, Chew P, et al. The safety and efficacy of brinzolamide $1 \%$ /timolol $0.5 \%$ fixed combination versus dorzolamide $2 \%$ /timolol $0.5 \%$ in patients with open-angle glaucoma or ocular hypertension. J Glaucoma. 2009;18(4):293-300.

29. Sakai H, Shinjyo S, Nakamura Y, Nakamura Y, Ishikawa S, Sawaguchi S. Comparison of latanoprost monotherapy and combined therapy of $0.5 \%$ timolol and $1 \%$ dorzolamide in chronic primary angle-closure glaucoma (CACG) in Japanese patients. J Ocul Pharmacol Ther. 2005;21(6):483-489.

30. Silver LH. Clinical efficacy and safety of brinzolamide (Azopt), a new topical carbonic anhydrase inhibitor for primary open-angle glaucoma and ocular hypertension. Brinzolamide Primary Therapy Study Group. Am J Ophthalmol. 1998;126(3):400-408.

31. van der Valk R, Webers CA, Schouten JS, Zeegers MP, Hendrikse F, Prins MH. Intraocular pressure-lowering effects of all commonly used glaucoma drugs: a meta-analysis of randomized clinical trials Ophthalmology. 2005;112(7):1177-1185.

32. Katz IM, Hubbard WA, Getson AJ, Gould AL. Intraocular pressure decrease in normal volunteers following timolol ophthalmic solution. Invest Ophthalmol. 1976;15(6):489-492.

33. International Federation of Pharmaceutical Manufacturers and Associations Medical Dictionary for Regulatory Activities MedDRA Version 14.1 2011. Available from: http://www.meddra.org/sites/default/ files/guidance/file/intguide_14_1_english.pdf. Accessed January 6, 2014.
34. Michaud JE, Friren B. International Brinzolamide Adjunctive Study Group. Comparison of topical brinzolamide $1 \%$ and dorzolamide $2 \%$ eye drops given twice daily in addition to timolol $0.5 \%$ in patients with primary open-angle glaucoma or ocular hypertension. Am J Ophthalmol. 2001;132(2):235-243.

35. Sanseau A, Sampaolesi J, Suzuki ER Jr, Lopes JF, Borel H. Preference for a fixed combination of brinzolamide/timolol versus dorzolamide/timolol among patients with open-angle glaucoma or ocular hypertension. Clin Ophthalmol. 2013;7:357-362.

36. Realini T, Weinreb RN, Wisniewski SR. Diurnal intraocular pressure patterns are not repeatable in the short term in healthy individuals. Ophthalmology. 2010;117(9):1700-1704.

37. Varma R, Hwang LJ, Grunden JW, Bean GW, Sultan MB. Assessing the efficacy of latanoprost vs timolol using an alternate efficacy parameter: the intervisit intraocular pressure range. Am J Ophthalmol. 2009;148(2):221-226.

38. Lanzl I, Raber T. Efficacy and tolerability of the fixed combination of brinzolamide $1 \%$ and timolol $0.5 \%$ in daily practice. Clin Ophthalmol. 2011;5:291-298.

39. Kitazawa Y, Araie M; Research Group on MK-0507A. [Double-masked, phase III comparative study of the combination ophthalmic solution of the $1 \%$ dorzolamide hydrochloride $/ 0.5 \%$ timolol maleate (MK-0507A) in patients with glaucoma and ocular hypertension]. Nihon Ganka Gakkai Zasshi. 2011;115(6):495-507. Japanese.

40. Kamei Y, Yamada H, Yoshihara A, Yoshikawa K, Matsubara M. [Influence on blurred vision after brinzolamide instillation]. Atarashii Ganka. 2012;29(7):1007-1012. Japanese.

41. Silver LH. Ocular comfort of brinzolamide $1.0 \%$ ophthalmic suspension compared with dorzolamide $2.0 \%$ ophthalmic solution: results from two multicenter comfort studies. Brinzolamide Comfort Study Group. Surv Ophthalmol. 2000;44 Supp1 2:S141-S145.

42. Stein JD, Kim DS, Niziol LM, et al. Differences in rates of glaucoma among Asian Americans and other racial groups, and among various Asian ethnic groups. Ophthalmology. 2011;118(6):1031-1037.
Clinical Ophthalmology

\section{Publish your work in this journal}

Clinical Ophthalmology is an international, peer-reviewed journal covering all subspecialties within ophthalmology. Key topics include: Optometry; Visual science; Pharmacology and drug therapy in eye diseases; Basic Sciences; Primary and Secondary eye care; Patient Safety and Quality of Care Improvements. This journal is indexed on

\section{Dovepress}

PubMed Central and CAS, and is the official journal of The Society of Clinical Ophthalmology (SCO). The manuscript management system is completely online and includes a very quick and fair peer-review system, which is all easy to use. Visit http://www.dovepress.com/ testimonials.php to read real quotes from published authors. 